\title{
The Effects Visual Feedback on Social Behavior during Decision Making Meetings
}

\author{
Merel Brandon ${ }^{1,2}$, Simon Epskamp ${ }^{1}$, Thomas de Groot ${ }^{1,2}$, Tim Franssen ${ }^{1}$, \\ Bart van Gennep ${ }^{1}$, and Thomas Visser ${ }^{1}$ \\ ${ }^{1}$ University of Twente, Human Media Interaction, P.O. Box 217, \\ 7500 AE Enschede, The Netherlands \\ ${ }^{2}$ T-Xchange, P.O. Box 1123, 7500 BC Enschede, The Netherlands \\ \{merelbrandon, simoneskamp, timfranssen, bartvangennep, \\ thomasvisser\} astudent. utwente.nl, \\ thomas.degrootatxchange.nl
}

\begin{abstract}
This paper describes the design and evaluation of a visualization that provides feedback for meeting participants on their social behavior (Social Mirror). Our Social Mirror provides feedback on participation level, interactivity level, and level of agreement. For the evaluation we conducted an experiment where two groups of four participants each took part in a meeting with and in a meeting without the Social Mirror. The results showed that the participants could easily extract information from the Social Mirror without being distracted from the topic of discussion during the meeting. Our results further suggest that the Social Mirror leads to changes in the social behavior of the participant; in particularly due to the agreement visualization. Moreover most participants prefer meetings with the presence of the Social Mirror.
\end{abstract}

Keywords: Meeting, collaboration, decision making process, social mirror, social visualization, social behavior, social feedback.

\section{Introduction}

Finding solutions for complex problem requires the sharing of knowledge between stakeholders in the decision-making process [1]. For that, meetings in which the stakeholders meet are very important. Unfortunately, meetings are often ineffective and inefficient. One of the reasons can be found in the social signals and social behavior between discussants that facilitate communication. People sometimes behave in an undesired manner during meetings (e.g. aggressively dominating, not participating because of shyness or expression of negative feelings, indecisiveness) [2]. Dominant alternations of speaker turns between two meeting participants often occur during group meetings, leaving little opportunity for other participants to take the turn [3]. One of the pitfalls of meetings is social loafing: "people expend less effort when working in groups than working alone" [4]. Another risk of meetings is groupthink: the tendency of groups to strive for unanimity, leaving no space for nonconsensus thinking [5]. 
This paper describes the design and evaluation of a Social Mirror. A Social Mirror is a near real-time visualization that shows the discussants information about the social behavior of themselves and other people, and allows the discussants to recognize patterns in their social behavior over time [6]. Social signals and social behavior are expressions of a person's affective, attitudinal, or cognitive state towards social situations or interplay trough a multiplicity of non-verbal behavioral cues. Social signals are short intentional non-verbal expressions (e.g. turn taking or mirroring), social behavior last longer (e.g. agreement, politeness, empathy) [7]. Interestingly, previous research shows that feedback on social behavior could lead to improved group performance and overall effectiveness [8]. For example, visualizing the speaking time of participants of a group meeting has a balancing effect on their participation level during a meeting $[3,6,9,10]$.

We developed a visualization that provides feedback on three social aspects: participation level, interactivity level, and level of agreement. These aspects are considered determinants of social loafing, undesirable social behavior of participants (e.g. dominance, under participation, and indecisiveness), and dominant speaker alternation. In addition, agreement visualization is an indicator of groupthinking. The goal of our Social Mirror is to make the social behavior of participants explicit. We expect that this will motivate people to change their social behavior during a meeting. Through a user study we evaluated the effect of our Social Mirror on the social behavior of participants. We have explored the usability, perceived usefulness, and satisfaction of the participants with the Social Mirror.

The outline of the paper is as follows. We start by describing more related work on social visualizations. Next, we portray our Social Mirror system design. Further, we describe the evaluation method. Section four, five and six will describe respectively the research method, results, and the discussion of the results. Finally, we conclude this paper with our ideas for future research.

\section{Related Work}

Already in 1959 Smith and Kight [8] showed that giving feedback to meeting participants on their social behavior has a positive effect on productivity. Their experimental study showed that groups that received social behavior feedback between two meetings were significantly more productive during the second meeting as compared to the groups that were not given feedback.

Over the last decade several research projects focused on applications that provide automatic visual social behavioral feedback during meetings. The findings of these projects $[3,6,9,10]$ indicate that visualizing speaking length has a balancing effect on the level of participation: over-participators speak less, and under participators speak more.

The Meeting Mediator of Kim et al. [9] used Social Metric Badges to collect information about the participation level and the interactivity level. This information was provided to each participant on a mobile phone. The Meeting Mediator had significant balancing effect on both the participation level and on the interactivity level. 
The term Social Mirror comes from a paper by Karahalios and Bergstorm [6]. They describe two types of social mirrors we will briefly discuss here: the Conversational Clock and the Conversational Votes. The Conversation Clock is a shared display that shows the participants their turn taking history of the whole conversation. Participants can see who has been speaking when, for how long, and how loud. After testing this application with a small group they noticed that participants discovered patterns in the conversational flow. What's more, participants made inferences from these patterns. For example, they could recognize leader-follower roles. The Conversational Votes provide participants with a red and a green button to indicate their agreement with the speaker. The buttons were placed underneath the table, to make it possible to vote anonymously. The speaker could see in the visualization the amount of agreement or disagreement among the listeners. This resulted in speakers lengthening their turn when they saw that others disagreed. Despite this undesired result, Karahalios and Bergstrom described some preliminary interesting benefits of displaying agreement, namely an increased feeling of inclusion and satisfaction that all could express their opinion. To make increase this effect, they suggested a more detailed expression of the level of disagreement and agreement.

We have followed the suggestion of Karahalios and Bergstrom by supporting gradual agreement visualization.

\section{Social Mirror System}

The goal of our Social Mirror is to motivate meeting participants to behave socially in a desirable manner (e.g. actively participating, and not dominating the discussion) during meetings. We believed that making participants more aware of their behavior, and making their behavior more explicit to them and the group would motivate them to change their social behavior towards more desirable behavior. The system shouldn't make high-level inferences (e.g. dominance or participation level), because we believed that this would lead to false inferences, and participants' refusal of the system. Therefore, the system should visualize the relative speaking duration of each participant as an indicator of participation level and dominance. Speaking less than other participants doesn't necessarily mean that the participant is not actively participating. Whether social behavioral changes are desired when the system shows that a participant has spoken rarely, is up to the participant. The system provides participants insights in dominant speaker alternations by visualizing how long and how often pairs of participants have spoken following each other. Furthermore, we wanted to increase the feeling of inclusion and satisfaction by visualizing the level of agreement. By providing the participants with an interface to actively indicate their opinion about statements of others or meeting outcomes, we wanted to decrease indecisiveness.

The Social Mirror we designed uses two methods to collect information on the social behavior of the discussants: 1) agreement through a user interface, and 2) interactivity level and participation level through automatic speaker detection. 


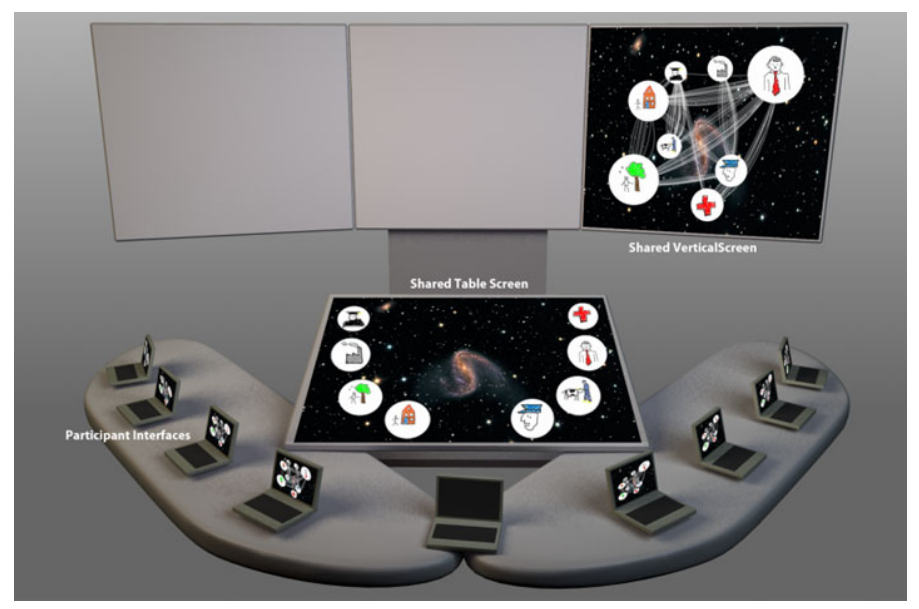

Fig. 1. The setup of the Social Mirror for eight participants

Fig. 1 shows the design of the system. Participants have their own user interface on a tablet $\mathrm{P}$. This interface allows them to draw their own avatar before the discussion starts. These avatars are used to represent the participants' alter ego on shared screens. We reasoned that it would be easier for participants to recognize a visual representation of themselves when they created it, than when it would be created for them. The visualization on the shared table-screen shows which avatar belongs to whom. The avatars are positioned in front of the participants. The interactive visualization on the shared vertical screen provides information about the social behavior of the participants. Fig. 2 shows a snapshot of the shared vertical screen during a group discussion. The participants have a similar view on their tablet PCs. The participants can express their agreement on the current discussion topic by controlling the position of their avatar via their tablet PC. A smaller distance between avatars signals more agreement, while a large distance indicates disagreement. The background image of the Social Mirror is normally the galaxy map as shown in fig. 2 . The facilitator (e.g. the moderator of the group discussion) can decide to change the background image. We have created two extra types of background images. First, a background image that has four diverse colors in the corners. This background can be used in case the meeting has four possible meeting outcomes. The participants can place their avatar on or in between the colors that represent the meeting outcome they prefer. Secondly, a gradient background image that is red on one side and green on the other. This background image can be used to indicate satisfaction with a solution, meeting outcome, or agreement with a statement.

The size of circles around the avatars indicates how much each participant has spoken during the discussion. Participants that are represented with bigger circles have spoken more during the meeting. 
The lines between the circles show how much the participants have spoken following one another. At short intervals a new line is drawn from the previous speaker to the current speaker.

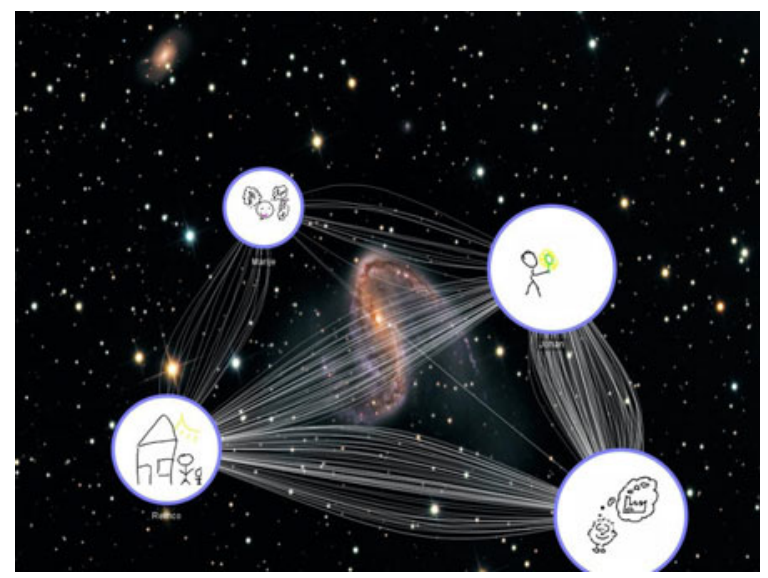

Fig. 2. The Social Mirror of four participants as displayed on the shared vertical screen and on the tablet PC. Distance between avatars signals level of agreement, size of avatars signals participation level, and lines between avatars signals interactivity level between pairs of participants.

\section{Evaluation}

The evaluation goal of this project was to explore the effects of the Social Mirror on social behavior of participants. We wanted insights in the usability, perceived usefulness, and satisfaction of the users with the system. We conducted a within subject design user study with two groups of four participants each. Qualitative data was collected through questionnaires. Both groups had a discussion with the Social Mirror (experimental condition), and a similar discussion without the Social Mirror (control condition). To eliminate learning effects, the order of the conditions was counterbalanced for the two groups. In both conditions participants were given similar tasks. Eight information engineering students participated ( 7 male, 1 female, average age 23). The experiment took place in a smart meeting room of the University of Twente. During both sessions there was a facilitator present, who led the meeting, and a second person that captured the content of the meeting in a mind map.

The participants were told that the goal of the experiment was to test a newly designed tool called the Social Mirror. After this introduction the first questionnaire was given with some personal data questions (e.g. age, gender, personality statements related to meeting behavior) and a consent form was signed. This personal data is used to check the similarity of the groups.

During each condition the participants were given a different (though similar) decision task (fictional but based on reality). During both tasks the group had to 
decide between four concepts for the site planning of a former airport terrain. All participants had been assigned a stakeholder role (nature lover, local government, resident, and businessman). The roles were not changed between conditions.

The interface of the Social Mirror was shortly explained to the participants. Though, the participants were not instructed with respect to the interpretation of, or the reaction to (e.g. desirable changes in their 'social' behavior) the Social Mirror.

During the experimental condition, participants were asked to draw their own avatar that would be used by the Social Mirror. As a starter, the facilitator asked the participants to indicate their position towards the four site planning concepts (e.g. which concept(s) they preferred). During the experimental condition the participants didn't only verbalized their position, but also visualized their position using the Social Mirror with four background colors that symbolized the four concepts. During the discussion with the Social Mirror the participants could freely move their avatar to signal their agreement with the other participants. Five minutes before the end of the meeting, the participants were asked to give their position towards the four concepts again. After the discussion that would last for 15 minutes they had to decide in favor of one of the four site planning concepts. The session ended with an assessment of the participants' satisfaction with the final decision. During the experimental condition the participants visualized their satisfaction using the green-to-red gradient background of the Social Mirror.

After each discussion (experimental and control) the participants completed a second questionnaire. These questionnaires asked the participants to score the social behavior of themselves and others. The questionnaire after the experimental condition had additional questions about the Social Mirror. After the participant experienced both conditions they were asked to indicate their preference for a meeting with or without the Social Mirror. The experiment ended with an informal debriefing with all participants.

\section{Evaluation Results}

\subsection{Social Behavior}

We asked the participants to score their own and each other's social behavior on a five point Likert scale with semantic labels after both the discussion with and the discussion without the Social Mirror. The participants scored how often each participant took the turn $(5=$ very frequently, $1=$ never $)$, for how long they spoke on average $(5=$ very long, $1=$ very short $)$, and how dominant they were $(5=$ very dominant, $1=$ very submissive). For each participant we have their own score and the group score (mean of the scores of others). Fig. 3 shows the mean and standard deviation of the self scores and group scores for social behavior. The difference between the mean scores for both conditions is very small. All scores are between three and four. The standard deviations are larger for the scores that are given after the discussion with the Social Mirror, except for the self assessment scores for dominance. 


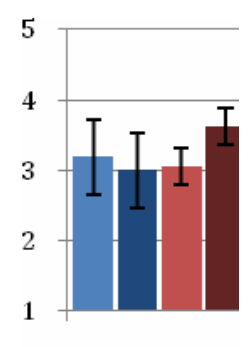

turn freq.

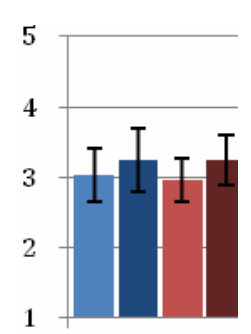

turn length

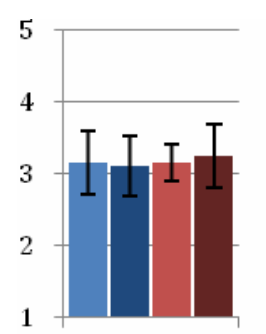

dominance

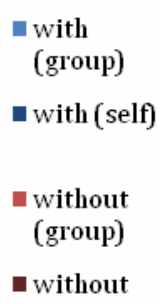

(self)

Fig. 3. Means and standard deviations of the scores that the participants gave themselves (self) and each other (group = mean group score of the social behavior of a participant) on the social behaviors (turn frequency, speaking length, and dominance) after the discussion with (with) and without (without) the Social Mirror

After the meeting with the Social Mirror the participants were given the following question: Has this Social Mirror influenced your behavior, how and why?

Seven out of eight participants felt that their social behavior was influenced by the Social Mirror. Most changes in the social behavior were related to the visualization of agreement. One answer was related to the participation level and one to the distribution of attention among the participants.

- "Yes, because you continuously have to indicate your position in relation to the other participants. You are forced to think about who you agree with."

- "It invited me to enter the discussion; you think more about your opinion."

- "I could clearly see to which people I should defend myself."

- "You are nudged to make more compromises (bad for your own view, good for the decision making)"

- "I felt more confident when someone agreed with me and dared to defend my view better."

- "I felt forced to talk when I saw my avatar being the smallest."

- "It made it clear who I was focusing on, do I could determine if I needed to give one of the participants more attention."

Only one participant said that the Social Mirror didn't have an effect on his behavior during the meeting. "Without this tool I would not behave differently, I would use the same arguments.”

\subsection{Usability}

The participants were asked to rate the statements about the usability of the Social Mirror on a five point Likert scale with semantic labels. Fig. 4. shows a diagram of the mean scores of agreement with the statements ( $1=$ strongly disagree, $5=$ strongly agree). 


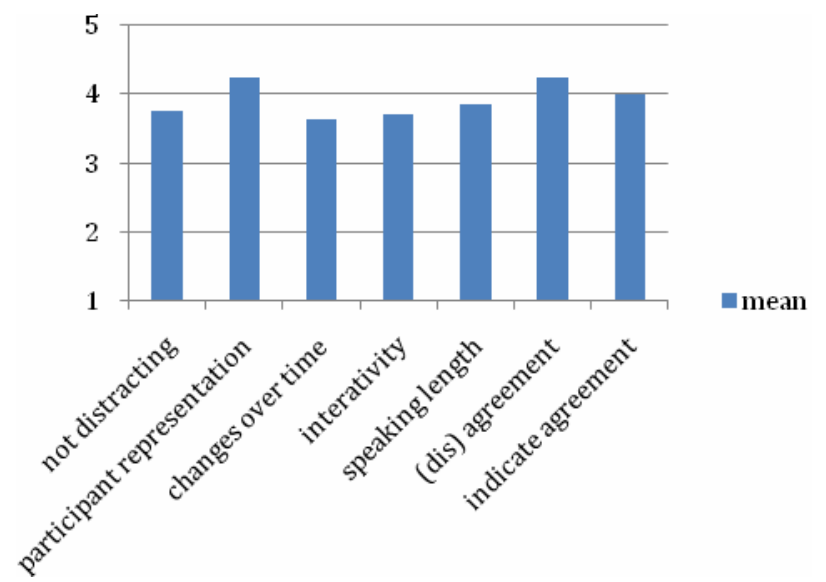

Fig. 4. Mean scores of participants' agreement with statements about the ease of understanding or using diverse aspects of the Social Mirror, and about how distractive the Social Mirror was perceived. Higher scores mean less distractive, easier to understand, or easier to use.

The answers to the open question: "What information did you get from the Social Mirror?," showed that the participants could read some additional information from the Social Mirror. They could see to what solution the discussion was heading, and the effect of arguments on other participants.

Six participants judged the appearance of the system as pretty, good, and useful. They liked drawing their own avatars. The gradient background image that visualized the four concepts as colors in the corners was perceived as a little boring and unclear. The galaxy map was perceived as pretty, but not useful.

\subsection{Preferences}

After both discussions the participants were given the following question; What do you prefer: a discussion with or without the Social Mirror? Seven out of eight participants preferred the meeting with the Social Mirror. All the motivations for the preference for the meeting with the Social Mirror were related to the visualization of the agreement. "It makes the views of participants much more clear." "When you weren't paying attention, you can quickly see what everyone's position is." "Consensus would become clear sooner visually." "You can see what moves people to change their view." The participants also state that the Social Mirror would be even more valuable during meetings with six or more participants.

The participant that preferred the meeting without the Social Mirror provided the following motivation. "I feel judged by the system. It gives me the feeling that I cannot take an extreme viewpoint.”

The facilitator also preferred the meeting with the Social Mirror. "It provided the facilitator more insights into the preference or view of participants... This makes it easier to work towards a decision. Participants see their own extreme views, and the facilitator does not have to point it out to them." 


\section{Discussion}

Our preliminary results show that our Social Mirror didn't have an effect on the speaking frequency, speaking length, or dominance of the participants. We measured these social behaviors by asking the participants to score themselves and each other on these aspects. This is a subjective and qualitative measurement of the social behavior and not fully reliable. In contrast to the related work we found more balance in the participation level during the meeting without the Social Mirror (the standard deviations of the scores of the control condition are smaller).

Most participants said that they changed their behavior during the meeting due to the presence of the Social Mirror. Most changes were related to the visualization of agreement. Participants were motivated to form an opinion, to reach consensus, actively participate, and equally divide their attention over all participants. Some of the changes that the participants described are behavioral changes but not social behavioral changes. During this explorative evaluation we used qualitative subjective measures of social behavioral changes. Naturally, there is always a bias between how people judge their own or each other's social behavior compared to their real social behavior.

The usability of the system was in general good. Most participants could easily read the Social Mirror without being distracted from the content of the meeting. However, participants forgot the meaning of the different colors of gradient background image. We told the participants that we created the Social Mirror. Therefore, participants could have given socially desirable answers. We doubt whether this is true in this case, since the questionnaires were anonymous and the answers to some other questions were sometimes quite negative. The level of distraction the system causes was only acquired through a subjective measurement.

Both participants and the facilitator preferred the meeting with the Social Mirror. Especially the visualization of agreement was perceived useful. The agreement visualization provided insights in the opinions of the participants, this allowed them to determine a persuasion strategy, to see when consensus was reached, and even predict meeting outcome. The answers didn't provide insight in the perceived usefulness of the visualization of the participation level and the interactivity level. The participant that didn't prefer the presence of the Social Mirror, didn't like the Social Mirror to make his extreme opinion explicit. He indicated that he felt forced to reach consensus. This finding could be explained in two ways. First, the Social Mirror could lead to discomfort and group thinking (only consensus thinking). Second, visualizing agreement motivates participants to reach consensus. These interpretations are not exclusive.

The main shortcoming of this study is the small number of participants. Therefore, we can't formulate any strong conclusions.

\section{Future Work}

Our findings suggest that visualizing the level of agreement between participants is perceived as a useful feature during decision making meetings. We therefore suggest future research to explore the effect of a Social Mirror that visualizes agreement on 
social behavior of participants and on meeting performance. The effect of agreement visualization on participation level, group thinking, and consensus reaching should be further investigated during future work. These effects might be more salient during meetings with six to eight participants.

In contrast to related work we didn't find a balancing effect of the Social Mirror on the participation level. Contrary, we found more balance in the participation level during the meeting without the Social Mirror. We therefore suggest a more extensive study of what aspects of a Social Mirror motivates people to change their social behavior. We would suggest a larger scale experiment with objective quantitative measurements.

At last we would suggest exploring the effect of the Social Mirror on real life meetings, where outcomes have far reaching consequences. We expect different effects of the Social Mirror during those kinds of meetings.

Acknowledgments. We would like to thank M. Poel and F.W. Fikkert of the HMI group of the University of Twente, and J. de Heer of T-Xchange for supervising the project.

\section{References}

1. Conklin, J.: Dialogue Mapping: Building Shared Understanding of Wicked Problems (2005)

2. Wayne, D.: The IAF handbook of group facilitation. In: Facilitation. Beyond Methods, ch. 3, pp. 35-55 (2005)

3. Sturm, J., van Herwijnen, O.H., Eyck, A., Terken, J.: Influencing social dynamics in meetings through a peripheral display. In: Proceedings of the ninth international conference on Multimodal interfaces - ICMI 2007, p. 263 (2007)

4. Jackson, J.M., Harkins, S.G.: Equity in Effort: An Explanation of the Social Loafing Effect. Journal of Personality 49, 1199-1206 (1985)

5. Hensley, T.R., Griffin, G.W.: Victims of Groupthink. Journal of Conflict Resolution 30, 497-531 (2010)

6. Karahalios, K.G., Bergstrom, T.: Social mirrors as social signals: transforming audio into graphics. IEEE computer graphics and applications 29, 22-32 (2009)

7. Vinciarelli, A., Pantic, M., Bourlard, H.: Social signal processing: Survey of an emerging domain. Image and Vision Computing 27, 1743-1759 (2009)

8. Smith, E.E., Kight, S.S.: Effects of feedback on insight and problem solving efficiency in training groups. Journal of Applied Psychology 43, 209-211 (1959)

9. Kim, T., Pentland, A.S., Chang, A.: Meeting Mediator: Enhancing Group Collaboration using Sociometric Feedback. In: Proceedings of the 2008 ACM conference on Computer supported cooperative work- CSCW 2008, pp. 457-466 (2008)

10. DiMicco, J.M., Pandolfo, A., Bender, W.: Influencing group participation with a shared display. In: Proceedings of the 2004 ACM conference on Computer supported cooperative work - CSCW 2004, p. 614 (2004) 